PROCEEDINGS OF THE

AMERICAN MATHEMATICAL SOCIETY

Volume 127, Number 1, January 1999, Pages 235-239

S 0002-9939(99)04505-0

\title{
COMPLETE POSITIVITY OF ELEMENTARY OPERATORS
}

\author{
LI JIANKUI
}

(Communicated by Dale E. Alspach)

\begin{abstract}
In this paper, we prove that if $\mathcal{S}$ is an $n$-dimensional subspace of $L(H)$, then $\mathcal{S}$ is $\left(\left[\frac{n}{2}\right]+1\right)$-reflexive, where $\left[\frac{n}{2}\right]$ denotes the greatest integer not larger than $\frac{n}{2}$. By the result, we show that if $\Phi(\cdot)=\sum_{i=1}^{n} A_{i}(\cdot) B_{i}$ is an elementary operator on a $C^{*}$-algebra $\mathcal{A}$, then $\Phi$ is completely positive if and only if $\Phi$ is $\left(\left[\frac{n-1}{2}\right]+1\right)$-positive.
\end{abstract}

In this paper, let $\mathrm{H}$ denote a complex Hilbert space. Let $H^{(n)}$ denote the direct sum of $n$ copies of $H$. For $T \in L(H)$, we write $T^{(n)}$ for the operator on $H^{(n)}$ which is the direct sum of $n$ copies of $T$; the notation is extended to a subset of $L(H)$ by $\mathcal{S}^{(n)} \equiv\left\{T^{(n)} \in L\left(H^{(n)}\right): T \in \mathcal{S}\right\}$. If $\mathcal{S}$ is a subspace of $L(H), \mathcal{S}$ is called $n$-reflexive if $\mathcal{S}^{(n)}=\operatorname{ref}\left(\mathcal{S}^{(n)}\right) \equiv\left\{T^{(n)} \in L\left(H^{(n)}\right): T^{(n)} x \in\left[\mathcal{S}^{(n)} x\right]\right.$, for all $\left.x \in H^{(n)}\right\}$, where [.] denotes norm closed linear span. By the definiton, we have that if $\mathcal{S}$ is $m$-reflexive, then $\mathcal{S}$ is $n$-reflexive for $n \geq m$. A separating vector for a subspace $\mathcal{S}$ of $L(H)$ is a vector $x \in H$ such that $T \mapsto T x, T \in \mathcal{S}$, is an injective map. For $x, y \in H$, let $x \otimes y$ denote the rank-one operator $u \mapsto(u, x) y$.

Let $\mathcal{A}$ denote a $C^{*}$-algebra. Then $\mathcal{A}$ is called primitive, if $\mathcal{A}$ has a faithful irreducible representation on some Hilbert space. An elementary operator $\Psi$ on $\mathcal{A}$ is a linear mapping of the form $\Psi: T \mapsto \sum_{i=1}^{n} A_{i} T B_{i}$, where $\left\{A_{i}\right\}_{i=1}^{n}$ and $\left\{B_{i}\right\}_{i=1}^{n}$ are subsets of $\mathcal{A}$. In this paper, we assume that all elementary operators are nonzero. A linear map $\Phi$ on $\mathcal{A}$ is positive (resp. hermitian-preserving) if $\Phi(T)$ is positive (resp. hermitian) for all positive (resp. hermitian) $T$ in $\mathcal{A}$. We define $\Phi_{n}=\Phi \otimes I_{n}$ : $M_{n}(\mathcal{A}) \rightarrow M_{n}(\mathcal{A})$ by $\Phi \otimes I_{n}\left(\left(T_{i j}\right)_{n \times n}\right)=\left(\Phi\left(T_{i j}\right)\right)_{n \times n}$. $\Phi$ is said to be $n$-positive if $\Phi \otimes I_{n}$ is positive. If $\Phi$ is $n$-positive for all $n$, then $\Phi$ is said to be completely positive.

In [4], Magajna states the following problem:

For each positive integer $r$ determine the smallest $k=k(r)$ such that all $r$ dimensional subspaces of $L(H)$ are $k$-reflexive.

In [4], Magajna proves $k \leq r$. In this paper, we prove that if $\mathcal{S}$ is an $n$-dimensional subspace of $L(H)$, then $\mathcal{S}$ is $\left(\left[\frac{n}{2}\right]+1\right)$-reflexive. Also by this result, we study complete positivity of elementary operators on a $C^{*}$-algebra $\mathcal{A}$. We prove that if $\Phi(\cdot)=\sum_{i=1}^{n} A_{i}(\cdot) B_{i}$ is an elementary operator on a $C^{*}$-algebra $\mathcal{A}$, then $\Phi$ is

Received by the editors July 8, 1996 and, in revised form, May 14, 1997.

1991 Mathematics Subject Classification. Primary 47B47, 47B49; Secondary 46L05.

Key words and phrases. Reflexivity, elementary operator, complete positivity.

(C)1999 American Mathematical Society 
completely positive if and only if $\Phi$ is $\left(\left[\frac{n-1}{2}\right]+1\right)$-positive. This result improves Theorem 2 [6].

Lemma 1. If the operators $A_{j}=a_{j} \otimes c(j=1, \ldots, n)$ form a basis of a subspace $\mathcal{S}$ of $L(H)$, then $\mathcal{S}$ is reflexive.

Proof. Let $T \in L(H)$ be such that for any $x \in H, T x \in[\mathcal{S} x]$, we have $T=b \otimes c$. If $x$ is orthogonal to all $a_{j}$, then $A_{j} x=0$ for all $j$. Thus $T x=0$ and $x$ must be orthogonal to $b$. This implies that $b \in \operatorname{span}\left\{a_{1}, \ldots, a_{n}\right\}$, hence $T \in \mathcal{S}$.

The following result is given in [2].

Lemma 2. Let $A, B \in L(H)$. Then $A x$ and $B x$ are linearly dependent for every $x$ in $L(H)$ if and only if one of the following conditions holds:

(i) $A$ and $B$ are linearly dependent;

(ii) there exist $x_{0}, x_{1}, x_{2} \in H$ with $A=x_{1} \otimes x_{0}$ and $B=x_{2} \otimes x_{0}$.

Theorem 3. If $\mathcal{S}$ is a subspace of $L(H)$ and $\operatorname{dim} \mathcal{S}=n$, then $\mathcal{S}$ is $\left(\left[\frac{n}{2}\right]+1\right)$ reflexive.

Proof. If $n=1,2$, by Magajna's result [4], then the theorem is true.

Suppose now, inductively, that the theorem is true for any subspace of $L(H)$ of dimension at most $n-1$, where $n \geq 3$, and let $\mathcal{S}$ be an $n$-dimensional subspace of $L(H)$. Let $\left\{A_{1}, \ldots, A_{n}\right\}$ be a basis of $\mathcal{S}$ and define

$$
k=\max \left\{\operatorname{dim} \operatorname{span}\left\{A_{1} x, \ldots, A_{n} x\right\}: x \in H\right\},
$$

then $1 \leq k \leq n$.

If $k=1$, by Lemmas 1 and 2, we have that the theorem is true.

If $k=n$, then $\operatorname{span}\left\{A_{1}, \ldots, A_{n}\right\}$ has a separating vector $x_{0}$. In the following, we prove that $\operatorname{span}\left\{A_{1}, \ldots, A_{n}\right\}$ is 2-reflexive. Suppose

$$
T^{(2)}\left(\begin{array}{l}
x \\
y
\end{array}\right) \in\left[\mathcal{S}^{(2)}\left(\begin{array}{l}
x \\
y
\end{array}\right)\right]
$$

for every pair $x, y$ of vectors in $H$. Then for each $y \in H$, there is a $T_{y} \in \mathcal{S}$ satisfying $T_{y} x_{0}=T x_{0}, T_{y} y=T y$. Since $x_{0}$ is separating for $\mathcal{S}, T_{y}$ must be independent of $y$, so $T \in \mathcal{S}$. By $n \geq 3$, we have $\left(\left[\frac{n}{2}\right]+1\right) \geq 2$. Hence $\mathcal{S}$ is $\left(\left[\frac{n}{2}\right]+1\right)$-reflexive.

If $2 \leq k \leq n-1$, we may assume, by reordering the $A_{i}$ if necessary, that there exists a vector $x_{0}$ such that $\left\{A_{i} x_{0}\right\}_{i=1}^{k}$ is linearly independent. Hence there is a unique $k \times(n-k)$ complex matrix $\left(a_{i j}\right)$ such that

$$
A_{k+j} x_{0}=\sum_{i=1}^{k} a_{i j} A_{i} x_{0}, j=1, \ldots, n-k .
$$

In the following, let $l=\left[\frac{n}{2}\right]+1$. If $A \in L(H)$, for any $x \in H^{(l)}$, satisfies $A^{(l)} x \in$ $\left[\mathcal{S}^{(l)} x\right]$, then for any $x_{1}, \ldots, x_{l-1}$ in $H$, there exist scalars $t_{1}, \ldots, t_{n}$ such that

$$
\left(\begin{array}{c}
A x_{0} \\
\vdots \\
A x_{l-1}
\end{array}\right)=t_{1}\left(\begin{array}{c}
A_{1} x_{0} \\
\vdots \\
A_{1} x_{l-1}
\end{array}\right)+\ldots+t_{n}\left(\begin{array}{c}
A_{n} x_{0} \\
\vdots \\
A_{n} x_{l-1}
\end{array}\right) \text {. }
$$


Since $A x_{0} \in \operatorname{span}\left\{A_{1} x_{0}, \ldots, A_{n} x_{0}\right\}=\operatorname{span}\left\{A_{1} x_{0}, \ldots, A_{k} x_{0}\right\}$, there exist scalars $v_{1}, \ldots, v_{k}$ such that

$$
A x_{0}=\sum_{i=1}^{k} v_{i} A_{i} x_{0}
$$

By (1), (2) and (3), we have that

$$
A x_{g}=\sum_{i=1}^{k}\left(v_{i}-\sum_{j=1}^{n-k} t_{j+k} a_{i j}\right) A_{i} x_{g}+\sum_{j=1}^{n-k} t_{j+k} A_{k+j} x_{g}, g=1, \ldots, l-1 .
$$

Let

$$
C=A-\sum_{i=1}^{k} v_{i} A_{i} \text { and } B_{j}=A_{k+j}-\sum_{i=1}^{k} a_{i j} A_{i}, j=1, \ldots, n-k .
$$

For any $x_{1}, \ldots, x_{l-1}$, by (2), (4) and (5), we have

$$
\left(\begin{array}{c}
C x_{1} \\
\vdots \\
C x_{l-1}
\end{array}\right)=t_{k+1}\left(\begin{array}{c}
B_{1} x_{1} \\
\vdots \\
B_{1} x_{l-1}
\end{array}\right)+\ldots+t_{n}\left(\begin{array}{c}
B_{n-k} x_{1} \\
\vdots \\
B_{n-k} x_{l-1}
\end{array}\right) .
$$

Since $2 \leq k \leq n-1$ and $n-k \leq n-2$, we have $l-1 \geq\left[\frac{n-k}{2}\right]+1$. By the inductive hypothesis, we have $C \in \operatorname{span}\left\{B_{1}, \ldots, B_{n-k}\right\}$, hence $A \in \operatorname{span}\left\{A_{1}, \ldots, A_{n}\right\}$.

Corollary 4. Let $\mathcal{S}$ be as in Theorem 5. If $\operatorname{dim} \mathcal{S}_{F}=m$, where $\mathcal{S}_{F}$ denotes all finite-rank operators in $\mathcal{S}$, then $\mathcal{S}$ is $\left(\left[\frac{m}{2}\right]+1\right)$-reflexive.

Proof. By Theorem 2.6 [3], we have that $\operatorname{ref}\left(\mathcal{S}^{(n)}\right)=\mathcal{S}^{(n)}+\operatorname{ref}\left(\mathcal{S}_{F}^{(n)}\right)$. Hence $\mathcal{S}^{(n)}$ is reflexive if and only if $\mathcal{S}_{F}^{(n)}$ is reflexive. By Theorem 3, it follows that $\mathcal{S}$ is $\left(\left[\frac{m}{2}\right]+1\right)$-reflexive.

Remark. By Theorem 3, a routine modification of the proof of Proposition 4.3 [4], we may prove that for any $r$-dimensional subspace $\mathcal{S}$ of a countably generated von Neumann algebra $\mathcal{R}$ on a Hilbert space $H$ the space $\overline{\mathcal{R}^{\prime} \mathcal{S}}$ is $\left(\left[\frac{r}{2}\right]+1\right)$-reflexive (relative to $\mathrm{L}(\mathrm{H})$ ), and the space $\overline{\varepsilon \mathcal{S}}$ is $\left(\left[\frac{r}{2}\right]+1\right)$-reflexive relative to $\mathcal{R}$ where $\varepsilon$ is the center of $\mathcal{R}$.

Lemma 5. If $\Phi(\cdot)=\sum_{i=1}^{n} A_{i}(\cdot) B_{i}$ is an elementary operator on $L(H)$, then $\Phi$ is completely positive if and only if $\Phi$ is $\left(\left[\frac{n-1}{2}\right]+1\right)$-positive.

Proof. The necessity is obvious, we only need to prove the sufficiency.

We may assume that $\left\{A_{1}, \ldots, A_{n}\right\}$ and $\left\{B_{1}, \ldots, B_{n}\right\}$ are linearly independent. Since $\Phi$ is positive, it follows that $\Phi$ is a hermitian-preserving elementary operator. By Corollary 4.9 [5], we have

$$
\Phi(\cdot)=\sum_{i=1}^{k} D_{i}(\cdot) D_{i}^{*}-\sum_{i=k+1}^{n} D_{i}(\cdot) D_{i}^{*},
$$

where $\left\{D_{i}\right\}_{i=1}^{n}$ is linearly independent.

To prove that $\Phi$ is completely positive, it suffices to prove $k=n$. If $n=1$, by (6), since $\Phi$ is positive, then the lemma is true. In the following, let $m=\left[\frac{n-1}{2}\right]+1$. 
If $n>1$ and $k \leq n-1$, since $\Phi$ is $m$-positive, for any vectors $x$ and $y$ in $H^{(m)}$, we have

$$
\left\langle\left(\Phi_{m}(x \otimes x)\right) y, y\right\rangle=\sum_{i=1}^{k}\left|\left\langle D_{i}^{(m)} x, y\right\rangle\right|^{2}-\sum_{i=k+1}^{n}\left|\left\langle D_{i}^{(m)} x, y\right\rangle\right|^{2} \geq 0 .
$$

By (7), we have that

$$
D_{j}^{(m)} x \in \operatorname{span}\left\{D_{1}^{(m)} x, \ldots, D_{k}^{(m)} x\right\}, k+1 \leq j \leq n .
$$

Since $1 \leq k \leq n-1$, by Theorem 3 , we have that $\operatorname{span}\left\{D_{1}, \ldots, D_{k}\right\}$ is $\left(\left[\frac{k}{2}\right]+1\right)$ reflexive. Since $\left[\frac{k}{2}\right]+1 \leq\left[\frac{n-1}{2}\right]+1$, we have that $\operatorname{span}\left\{D_{1}, \ldots, D_{k}\right\}$ is $m$-reflexive. By (8), we have that $D_{k+1}, \ldots, D_{n}$ belongs to $\operatorname{span}\left\{D_{1}, \ldots, D_{k}\right\}$. Since $\left\{D_{i}\right\}_{i=1}^{n}$ is linearly independent, this is a contradiction.

Theorem 6. If $\Phi(\cdot)=\sum_{i=1}^{n} A_{i}(\cdot) B_{i}$ is an elementary operator on a $C^{*}$-algebra $\mathcal{A}$, then $\Phi$ is completely positive if and only if $\Phi$ is $\left(\left[\frac{n-1}{2}\right]+1\right)$-positive.

Lemma 7. Theorem 6 holds when $\mathcal{A}$ is primitive.

Proof. The necessity is trivial, so we have only to prove the sufficiency.

Since $\mathcal{A}$ is primitive, we may assume that $\mathcal{A}$ acts irreducibly on the Hilbert space $H$. By $\Phi$, we may induce an elementary operator $\tilde{\Phi}$ on $L(H)$ defined by $\tilde{\Phi}(T)=\sum_{i=1}^{n} A_{i} T B_{i}$ for any $T$ in $L(H)$. Since $\mathcal{A}$ is irreducible, we have that $\mathcal{A}$ is strongly dense in $L(H)$. Hence $\Phi$ is $m$-positive on $\mathcal{A}$ if and only if $\tilde{\Phi}$ is $m$-positive on $L(H)$. Since $\Phi$ is $\left(\left[\frac{n-1}{2}\right]+1\right)$-positive, we have that $\tilde{\Phi}$ is $\left(\left[\frac{n-1}{2}\right]+1\right)$-positive. By Lemma 5 , we have that $\tilde{\Phi}$ is completely positive. Hence $\Phi$ is completely positive.

Proof of Theorem 6. The forward implication is obvious.

Conversely, let $\pi$ be an irreducible representation of $\mathcal{A}$ on $H$. Then $\Phi$ induces an elementary operator $\pi \Phi(\cdot)=\sum_{i=1}^{n} \pi\left(A_{i}\right)(\cdot) \pi\left(B_{i}\right)$ on $L(H)$. By Lemma 7 , we have that $\pi \Phi$ is completely positive. Let $\pi_{a}=\bigoplus_{t \in \hat{A}} \pi_{t}$ be the reduced atomic representation of $\mathcal{A}$ on $H_{a}=\bigoplus_{t \in \hat{A}} H_{t}$. Then $\pi_{a}$ is a faithful representation of $\mathcal{A}$ on $H_{a}$. Since $\pi_{t} \Phi$ is completely positive on $L\left(H_{t}\right)$, we have $\pi_{a} \Phi$ is completely positive on $\prod_{t \in \hat{A}} L\left(H_{t}\right)$. Since $\pi_{a}$ is a faithful representation of $\mathcal{A}$ on $L\left(H_{a}\right)$, we have that $\Phi$ is completely positive.

Remark. Theorem 6 improves Theorem $2[6]$ which gives that if $\Phi$ and $\mathcal{A}$ are as in Theorem 6 , then $\Phi$ is $n$-positive if and only if $\Phi$ is completely positive.

\section{NOTE ADDED IN PROOF}

Recently Z. Pan and the author resolved Magajna's problem [4]. We prove that if $\mathcal{S}$ is an $n$-dimensional subspace of $L(H)$, then $\mathcal{S}$ is $[\sqrt{2 n}]$-reflexive and $k(n)=[\sqrt{2 n}]$.

\section{ACKNOWLEDGEMENTS}

The author wishes to thank the referee for useful suggestions. 


\section{REFERENCES}

1. J. Hou, On operator inequalities and combinations of operators, Linear Alg. Appl. 153 (1991), 35-51. MR 92e:47060

2. J. Hou, Linear interpolations and elementary operators on $B(H)$, Sci. China, Ser. A 36 (1993), 1025-1035. MR 95f:47034

3. D. R. Larson, Reflexivity, algebraic reflexivity and linear interpolation, Amer. J. Math. 110 (1988), 283-299. MR 89d:47096

4. B. Magajna, On the relative reflexivity of finitely generated modules of operators, Tran. Amer. Math. Soc. 327 (1991), 221-249. MR 91m:47064

5. M. Mathieu, Elementary operators on prime $C^{*}$-algebras I, Math. Ann. 284 (1989), 223-244. MR 90h: 46092

6. M. Mathieu, How to use primeness to describle properties of elementary operators, Proc. Symp. Pure Math. part 2, 51 (1990), 195-199. CMP 91:03

7. G. K. Pederson, $C^{*}$-Algebras and their Automorphism Groups, Academic Press, London, 1979.

Department of Mathematics, Hunan Normal University, Changsha, Hunan 410081, People's Republic of China

Current address: Department of Mathematics, University of New Hampshire, Durham, New Hampshire 03824

E-mail address: jkli@spicerack.sr.unh.edu 